\title{
Structure and Activity of the DHNA Coenzyme-A Thioesterase from Staphylococcus aureus Providing Insights for Innovative Drug Development
}

\author{
Aline Melro Murad \\ University Hamburg \\ Hévila Brognaro \\ University Hamburg \\ Sven Falke \\ University Hamburg \\ Jasmin Lindner \\ University of São Paulo \\ Markus Perbandt \\ University Hamburg \\ Celestin Mudogo \\ University Hamburg \\ Robin Schubert \\ University Hamburg \\ Carsten Wrenger \\ University of São Paulo \\ Christian Betzel ( $\nabla$ christian.betzel@uni-hamburg.de) \\ University Hamburg
}

\section{Research Article}

Keywords: MRSA, drug discovery, vitamin K2 pathway, DHNA-CoA thioesterase

Posted Date: October 27th, 2021

DOI: https://doi.org/10.21203/rs.3.rs-965646/v1

License: (1) (i) This work is licensed under a Creative Commons Attribution 4.0 International License.

Read Full License

Version of Record: A version of this preprint was published at Scientific Reports on March 12th, 2022. See the published version at https://doi.org/10.1038/s41598-022-08281-2. 


\section{Abstract}

The humanity is facing an increasing health threat caused by a variety of multidrug resistance bacteria. Within this scenario, Staphylococcus aureus, in particular methicillin resistant $S$. aureus (MRSA), is responsible for a number of hospital-acquired bacterial infections. The emergence of microbial antibiotic resistance consequently urgently demands to identify new and innovative strategies to treat antibiotic resistant microorganisms. In this context, structure and function analysis of potential drug targets in metabolic pathways vital for bacteria endurance, such as the vitamin $\mathrm{K}_{2}$ synthesis pathway, becomes interesting. We have solved and refined the crystal structure of the $S$. aureus DHNA thioesterase (SaDHNA), a key enzyme in the vitamin $\mathrm{K}_{2}$ pathway. The crystallographic structure in combination with small angle X-ray solution scattering data revealed a functional tetramer of SaDHNA. Complementary activity assays of SaDHNA indicated a preference for hydrolysing long acyl chains. Site-directed mutagenesis of SaDHNA confirmed the functional importance of Asp16 and Glu31 for thioesterase activity and substrate binding at the putative active site, respectively. The high-resolution structure of SaDHNA and complementary information about substrate binding will support future drug discovery and design investigations to inhibit the vitamin $\mathrm{K}_{2}$ synthesis pathway, vital for the bacteria.

\section{Introduction}

The increase in hospital infections $(\mathrm{HI})$ remains to be one of the major concerns in the global health system. Bacterial infections acquired during patient hospitalization contribute not only to significant mortality, but also usually require additional therapies, increasing even more the financial burden for the healthcare systems ${ }^{1}$. Among bacterial infections involved in $\mathrm{HI}$, Staphylococcus aureus is both commensal and a leading pathogen found in hospitals causing serious endocarditis, pneumonia, skin and wound infections ${ }^{2}$. The first successful treatment of $S$. aureus infections, in the 1940's, involved mainly the administration of $\beta$-lactam antibiotics like penicillin $\mathrm{G}$. Targeting the bacterial enzymes of the cell wall biosynthesis, called penicillin-binding proteins (PBPs), the inhibition of the PBPs induced by $\beta$ lactam antibiotics interferes with the cross-linking of peptidoglycan, making the cell wall mechanically fragile and the cell perish ${ }^{3}$. However, since penicillin resistance was first discovered in the early $1950 \mathrm{~s}$, due to the production of specific $\beta$-lactamases by the acquisition of the $m e c A$ gene, capable to hydrolyse $\beta$-lactam antibiotics, the phenomenon of drug resistance has been observed for an increasing number of Gram-negative and Gram-positive pathogens ${ }^{4}$. The development of methicillin, a semi synthetic antibiotic derivative, assigned to replace conventional penicillin treatment, raised the emergence of Methicillin resistant $S$. aureus (MRSA). Unfortunately, $S$. aureus currently exhibits resistance not only to methicillin but also against a number of antimicrobial agents, including carbapenems, cephalosporins ( $\beta$-lactam class) and even to last-line antibiotics such as linezolid and the glycopeptide vancomycin ${ }^{5}$. The threating increase of multidrug resistant $S$. aureus, in particular MRSA, has narrowed therapy options substantial, making the medical treatment of MRSA infections even more complex. In order to overcome bacterial resistance, identification of novel drug targets as well as the development of innovative antibiotics with high specificity and effectiveness needs to be enforced. Latest drug discovery investigations are focusing 
on obtaining structural knowledge about enzymes involved in the bacterial cell metabolism, which are critical and vital for bacteria endurance. The lipid-soluble compound vitamin $\mathrm{K}_{2}$ (menaquinone), with about $85-90 \%$ almost entirely located in the bacterial membrane, is an important electron carrying component in the membrane-bound complexes of the electron-transport chain in cell respiration ${ }^{6}$. As the electron transport chain in humans does not involve menaquinone, the electron transport chain in bacteria is an indispensable and essential component for ATP production and presumably has substantial potential for novel drug development investigations. Humans entirely depend on the uptake of vitamin $\mathrm{K}$ which is known to improve health in cardiovascular disease, chronic kidney disease and bone metabolism. In bacteria, several enzymes involved in menaquinone de novo biosynthesis already have already been analysed, e.g. Escherichia coli MenD ${ }^{7}$, Mycobacterium turbeculosis MenB ${ }^{8}$ and Pseudomonas 4-Hydroxybenzoyl- CoA thioesterase ${ }^{9}$. Based on structural information of SaMenE, the authors designed several o-succinylbenzoyl (OSB) secondary amine analogues (OSB-AMS) with high specificity and showing antimicrobial activity. Furthermore, the effect of these OSB-AMS on vitamin K levels of $S$. aureus can be assigned to a direct interference with menaquinone biosynthesis. According to Matarlo et al. (2015), the identification of a putative interaction of OBS-AMS and the arginine Arg222 of SaMenE, and corresponding mutagenesis studies confirmed the importance of Arg222 in substrate binding as well as inhibition mechanisms of SaMenE ${ }^{10}$. Furthermore, Choi and collaborators, also designed, synthesized and evaluated several inhibitors based on the MenA structure and revealed promising inhibitory activity with low minimum inhibitory concentrations (MICs) ranging from 1 to $8 \mu \mathrm{g}$ $\mathrm{mL}^{-1}$ against MRSA strains ${ }^{11}$. These observations and evidences undoubtedly highlight the significance of the menaquinone pathway in $S$. aureus endurance and demonstrates that structural investigations of involved key enzymes will support drug discovery investigations targeting this pathway. Being one essential enzyme of the menaquinone pathway of $S$. aureus, 1,4-dihydroxy-2-naphthoyl coenzyme A thioesterase - SaDHNA (E.C. 3.1.2.28) can be considered as a potential target enzyme for drug discovery investigations. In this context we solved and refined the tetrameric structure of DHNA from S. aureus to $1.3 \AA$ resolution, which in combination with site-directed mutagenesis allowed also to identify residues essential for thioesterase activity and substrate binding.

\section{Results}

\subsection{Three-dimensional structure of the tetrameric SaDHNA}

Crystals of the native protein belong to the space group $P 2_{1}$ with unit cell dimensions of $a=53.61, b=$ $90.66, c=75.38 \AA, a=\gamma=90$ and $\beta=92\left(^{\circ}\right)$ with four molecules in the asymmetric unit. The calculated Matthews coefficient is $2.4 \AA^{3} \mathrm{Da}^{-1}$, corresponding to a approx. solvent content of $48 \%$. The SaDHNA monomer comprehends 155 amino acids with a corresponding molecular weight of $18.1 \mathrm{kDa}$ and a theoretical pl of 5.5. The structure of the SaDHNA tetramer was refined to $R_{\text {work }} / R_{\text {free }}(\%)$ values of $17.8 / 19.8$, respectively. Data collection statistics and final refinement parameters are summarized in Table 1. The residues 156-165, corresponding to the strep-tag II at the C-terminus (SA-WSHPQFEK), as 
well as the residues DGIDSL at the C-terminus of chain B and D were disordered and not included in the final model. The atom coordinates of SaDHNA were deposited in the Protein Data Bank with pdb code 6FDG. 
Table 1

Data collection, processing and refinement statistics

Native data

Deriv. data

\section{Data collection*}

Beamline

Wavelength $(\AA)$

Space group

Unit-cell parameters

$a(\AA ̊)$

$b(\AA)$

$c(\AA)$

$a=\gamma\left({ }^{\circ}\right)$

$\beta\left(^{\circ}\right)$

Resolution range $(\AA)$

Completeness (\%)

$R_{\text {merge }}(\%)$

Multiplicity

$\nabla / / \sigma(I) \square$

\section{Refinement}

Resolution range $(\AA)$

Noof reflections used for

refinement

Rwork/ Rfree (\%)

Noof atoms

Protein

Water

Average $B$ value $\left(\AA^{2}\right)$

R.m.s.d
P13, PETRA III (DESY, Germany)

1.0332

$P 2_{1}$

53.61

90.78

75.38

90

92.0

75.33-1.3 (1.33-1.3)

$97.9(96.1)$

$4.0(123)$

$6.9(7.0)$

$19.9(2.0)$

75.3-1.3

164237

$17.8 / 19.8$

5483

314

23.6
P14, PETRA III (DESY, Germany)

1.072

$P 2_{1}$

55.20

90.90

74.80

90

90.8

55.29-2.00 (2.1-2.0)

$98.2(94.5)$

$11.5(89.8)$

$6.9(6.6)$

$12.7(2.7)$ 


\begin{tabular}{|lll|}
\hline & Native data & Deriv. data \\
\hline Bonds $(\AA)$ & 0.03 & \\
\hline Angles $\left({ }^{\circ}\right)$ & 2.86 \\
\hline Ramachandran plot & \\
\cline { 1 - 2 } Favoured regions (\%) & 98.4 \\
\hline Allowed regions $(\%)$ & 1.6 \\
\hline Disallowed region $(\%)$ & 0 \\
\hline *Numbers in parenthesis refer to the outer resolution shell. \\
\hline
\end{tabular}

The overall structure of the SaDHNA monomer and topology are illustrated in Fig. 1A and B, showing that SaDHNA has a high content of compact secondary structure elements ( $26 \%$ a-helix and $32 \% \beta$ strand) with a relatively extended five-stranded antiparallel $\beta$-sheet element and one parallel $\beta$-sheet segment in the sequential order 8-1-4-5-6-3, involving the residues Val146-lle148 ( $\beta 8$ ), Met1-Ala10 ( $\beta 1)$,

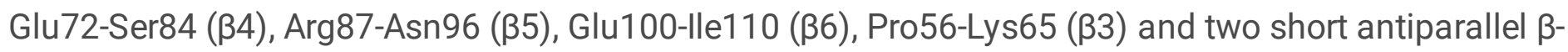
sheets containing the amino acid residues Gly52-lle54 ( $\beta 2$ ) and lle112-Glu. The $\beta$-sheets $\beta 5$ and $\beta 6$ are interrupted by four $\beta$-bulges at Val89, lle94, Asn96 and Glu108. All $\beta$-sheets wrap around the four $\alpha$ helices formed by residues Tyr26-Gly42 (a1), Ser44-Gln51 (a2), Arg121-Phe126 (a3) and Pro127-Glu142 (a4) and two short a-helices comprising the residues Arg11-Glu14 ( $₫ 1$ ) and Try22-Asn25 ( $\$ 2$ ) connected by a $\beta$-turn. The turns connecting the $\beta$-strands are type I (Ser84-Arg87, Asn96-Gly99) and type IV (Phe69Glu72, Lys113-Trr116). In addition, two $\beta$-hairpins between $\beta-4 / \beta-5$ (class $2: 4$ ) and $\beta-5 / \beta-6$ (class $3: 5$ ) and a $\gamma$-turn lle152-Ser154 after the last $\beta 8$ strand are observed within the structure.

Two monomers of SaDHNA assemble to form a homodimer by a network of nine hydrogen bonds between strand $\beta 3$ of chains $A$ or $C$ with the adjacent $\beta 3$ strand of chains $B$ or $D$, respectively, producing an overall 10-stranded antiparallel $\beta$-sheet motif involving the residues Thr58-Tyr64, Asp59-Asn63, Leu60Val62, Asn61-Asn61, Tyr64-Thr58, Asn63-Asp59 and Val62-Leu60. Three further H-bonds stabilize a2, a1 and $\varangle 2$, involving the residue pairs Glu49-Lys17, Glu31-Tyr22 and Tyr22-Glu31 respectively, along with two ionic interactions involving Lys17-Glu49. There are two hydrogen bonds between chains $\mathrm{A}$ and $\mathrm{C}$ stabilizing the loop involving Thr15 and Lys17. Between chains A and D four hydrogen bonds involving residues Arg11-Glu14, Glu14-Arg11, Tyr12-Glu49 stabilize interactions of $\nabla 1-\nabla 2$ and $\nabla 1-a 2$ and Glu31-

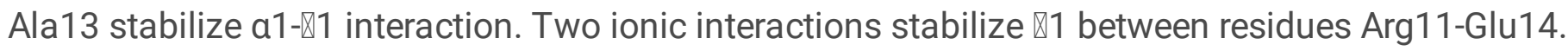
Additionally, the main a-helix (a1) is stabilized by a number of hydrophobic interactions. All SaDHNA chain interface features are summarized in Table 2. Thereby the quaternary structure of SaDHNA is formed by the assembly of four identical subunits $A, B, C$ and $D$, arranged as dimer of dimers, forming a homo-tetrameric structure, as shown in Fig. 1C. 
Table 2

Summary of SaDHNA chain interface features.

\begin{tabular}{|llllll|}
\hline Chains & $\begin{array}{l}\text { No. of residues located in } \\
\text { interface regions }\end{array}$ & $\begin{array}{l}\text { Interface } \\
\text { area }\left(\AA^{2}\right)\end{array}$ & $\begin{array}{l}\text { No. of } \\
\text { hydrogen } \\
\text { bonds }\end{array}$ & $\begin{array}{l}\text { No. of non- } \\
\text { bonded } \\
\text { contacts }\end{array}$ & $\begin{array}{l}\text { No. of } \\
\text { ionic } \\
\text { bonds }\end{array}$ \\
\hline A-B & $23: 20$ & $1051: 1070$ & 12 & 115 & 2 \\
\hline C-D & $22: 20$ & $1053: 1058$ & 12 & 121 & 1 \\
\hline A-C & $7: 8$ & $432: 432$ & 2 & 50 & - \\
\hline B-D & $7: 7$ & $428: 426$ & 4 & 52 & - \\
\hline A-D & $9: 9$ & $611: 621$ & 4 & 35 & 2 \\
\hline B-C & $10: 10$ & $614: 607$ & 4 & 38 & 2 \\
\hline
\end{tabular}

In the crystal structure four molecules of SaDHNA are present in the asymmetric unit. The oligomeric state analysis performed using PDBe PISA $^{12}$ provided a value for the buried area of each monomer at the A-B (or C-D) interface of approx. $1040 \AA^{2}$, corresponding to $13 \%$ of the overall surface area of each monomer. The program PBEQ solver ${ }^{13}$ was used to calculate the monomer/tetramer electrostatic surface potential to be $\Delta \mathrm{G}_{\text {elec }}$ of $-9516.320 \mathrm{kcal} \mathrm{mol}^{-1}$ for the tetramer and $-2676.08 \mathrm{kcal} \mathrm{mol}^{-1}$ for the monomer. In addition, the solvation energy was calculated to be $\Delta \Delta_{\text {elec }}$ of $6.840 \mathrm{kcal} \mathrm{mol}^{-1}$ by utilizing the PoissonBoltzmann equation ${ }^{14}$, indicating a significant increase in stability upon tetramer formation.

In order to verify the oligomeric state of SaDHNA in solution, we applied dynamic light scattering (DLS) and SAXS. DLS measurements showed a monodisperse hydrodynamic radius of $4.3 \pm 0.3 \mathrm{~nm}$ (Fig. 2) and SAXS data provided, based on the $p(r)$ function and using the Guinier approximation, a corresponding maximum diameter for SaDHNA of $10.9 \mathrm{~nm}$, and the radius of gyration was calculated to be $3.27 \pm 0.09 \mathrm{~nm}$. Those values along with the calculated SAXS ab initio model (Fig. 2C) confirm a functional tetramer in solution with a slightly elongated twisted "butterfly-like" shape, as observed also for the crystal structure.

\subsection{Comparison with homologues enzymes}

A search to identify and compare homologous structures was performed applying the European Bioinformatics Institute database server http://www.ebi.ac.uk/msd-srv/ssm. The top-scoring domain superimposition indicated homology to a hypothetical thioesterase (pdb code 1Z54) with a Ca core r.m.s.d. of $1.1 \AA$ for 41 aligned residues and $32 \%$ amino-acid sequence identity, along with another well characterized 4-hydroxybezoyl-CoA thioesterase from Pseudomonas sp. strain CBS3 (pdb code 1BVQ) ${ }^{9}$ with a corresponding Ca r.m.s.d of $1.7 \AA$ and $21 \%$ sequence identity. These results indeed confirm that SaDHNA belongs to the protein family displaying a HotDog domain fold class $I$. The structure is most conserved in $\beta 1$ and in strands $\beta 3$ up to $\beta 6$, in helix $\alpha 1$ and in the short a2 helix (Fig. 3A). The highest 
variability was observed in the loop region connecting $\beta 5$ and $\beta 6$ (Fig. 3A, light red box), as well as in the region connecting $\beta 6$ and $\alpha 4$ (Fig. $3 \mathrm{~A}$, light blue box). These structural differences can be assigned to the absence of two short antiparallel $\beta$ sheets in SaDHNA structure, as well as the presence of the extra ahelix $a 3$ and the elongated helix a4 including the residues Gln137-Lys144, the parallel strand $\beta 8$ (Val46Ile148) and a flexible loop (Met149-Leu155) at the C terminal region of SaDHNA. The protein sequence alignment of SaDHNA, shown in Fig. 3B, also revealed that Asp16 is an essential residue for a thioesterase activity (numbering according to SaDHNA). This residue is well conserved among the compared proteins.

\subsection{Putative active site of SaDHNA thioesterase}

For future drug discovery experiments targeting SaDHNA, detailed information about the location of putative active residues within the active site region and its interactions is an essential requirement. In order to obtain insights SaDHNA was superimposed to the D17N mutated 4-hydroxybenzoyl-CoA thioesterase from Pseudomonas sp strain $C B S-3^{16}$ (pdb code 1LO9) in complex with the substrate 4hydroxybenzoyl-CoA (BCA). It can be seen that a putative active site of SaDHNA is arranged by the interface region between two neighbouring monomers in the quaternary structure of a homo-tetramer, resulting in the formation of four active sites, as shown in Fig. 4A.

According to active site analysis investigations performed by Thoden et al. ${ }^{16}$, the binding of the ligand is mediated by interactions with the amino acid residues Tyr45 from one chain ( $B$ or $D$ ) and Asp16 and His23 from the corresponding neighbouring chain ( $A$ or $C$ ), all located in the interface region, as shown in Fig. 4B. According to the authors the binding of the substrate 4-hydroxybenzoyl CoA inside the binding pocket is supposed to be mainly stabilized by hydrogen bonds formed between the hydroxyl group from the aromatic moiety of the ligand with the benzoyl ring hydroxyl group of the amino acid residue Tyr45, as well as through the carbonyl carbon group from the amino acid Glu31 mediated by a water molecule. And, the BCA molecule side is positioned in a cavity located at the surface of one monomer and the remaining ligand is located in a deep cleft formed by the subunit-subunit interface. The position of the benzoyl ring hydroxyl group of the substrate may interact with the side chain hydroxyl group of Tyr45 from one monomer and with the sidechain of His23 located at helix $₫ 2$ from the corresponding subunit. Thereby the thioester carbonyl group of the substrate is located close to the $\mathrm{N}$-terminal region of a1, forming a hydrogen bond with the His23 side chain imidazole ring.

\subsection{Thioesterase activity assays and peptide binding analysis by fluorescence spectroscopy}

To obtain some initial information about SaDHNA substrate specificity of thioesterase, two different substrates classified according to its CoA chain length, one with a long acyl chain stearoyl-CoA (C18:0) and the other with a relative short chain crotonyl-CoA (C4:1). Thioesterase activity was identified and observed via the increase in free thiol-CoA thioester hydrolysis formation of 2-nitro-5-thiobenzoate anion $\left(\mathrm{TNB}^{2}\right)$, resultant of the reaction of thiolate anion $\left(\mathrm{RS}^{-}\right)$with Ellman's reagent $\left(\mathrm{DTNB}^{2-}\right)$ and one mixed disulphide (R-S-TNB ${ }^{-}$). SaDHNA showed 474-fold higher specific activity toward the extended acyl CoA 
chain in comparison to the short chain crotonyl-CoA (Supplemental Table S1). Furthermore, the significance of the amino acid residues Asp16 and Glu31 for thioesterase activity of SaDHNA was investigated applying the longer acyl CoA chain as substrate. The hydrolysis rate of stearoyl-CoA of the D16A mutant, which had the putative site carboxylate group removed, decreased 300-fold, while no activity was detected above the background level of stearoyl-CoA for the E31N mutant, indicating that both amino acid residues are essential for the activity of SaDHNA.

In parallel, in terms of structure-based computational design investigations, the atomic structure of SaDHNA was used for docking analysis applying the Bioluminate module from the Schrödinger suite, (Schrödinger, LLC, New York, 2021). Based on the structure of SaDHNA, and particular considering the putative active site (Asp16, His23, Glu31 and Try45) we designed several peptides (ranging from 5 to 6 residues) which potential can bind to SaDHNA and inhibit the activity. Docking investigations identified two binding sites, one at the SaDHNA surface and one in the putative active site. Among all, the most effective peptide inhibitors, named Pep-1 (YGSDGR) and Pep-2 (EGEYE), showed the smallest Optimized Potentials for Liquid Simulations (OPLS) force field (potential energy OPLS2005 -1583.93 kcal mol-1 and $-1927.2793 \mathrm{kcal} \mathrm{mol}^{-1}$, respectively $)^{17}$.

Pep-1 (YGSDGR), with a molecular weight of $654.28 \mathrm{Da}$, was designed and predicted to bind inside the active site region with a $\Delta \mathrm{G}_{\text {bind }}$ of $-81.0 \mathrm{kcal} \mathrm{mol}^{-1}$. According to the docking analysis, the protein-peptide complex is mediated mainly through six hydrogen bonds formed between Pep- 1 and residues present in the putative active site. The benzoyl ring of the tyrosine of Pep-1 has non-covalent $\pi$-stacking interactions with the Try45 benzoyl ring of the SaDHNA structure, as well as with the side chain of Ser55 through a hydrogen bond. The residues important for the substrate binding and activity, Glu31 and Asp16, respectively, are predicted to interact with the amide of the peptide backbone and with the side chain of serine of the peptide via hydrogen bonds as well. The second peptide (Pep-2) with a molecular weight of 623.23 $\mathrm{Da}$, was predicted not to interact with the residues in the putative active site region, but with residues located on the surface of the SaDHNA structure, close to the binding site entrance, with an $\Delta \mathrm{G}_{\text {bind }}$ of $-41.3 \mathrm{kcal} \cdot \mathrm{mol}^{-1}$. Pep-2 - SaDHNA interactions involve seven hydrogen bonds, as well as hydrophobic interactions between residues localized between connecting loops $\beta 2-\beta 3, \beta 4-\beta 5$ and $\beta 5$ - $\mathrm{a} 3$. In both cases, the predicted peptide binding free energies $\left(-81.0 \mathrm{kcal} \mathrm{mol}^{-1}\right.$ and $\Delta \mathrm{G}_{\text {bind }}$ of $-41.3 \mathrm{kcal} \mathrm{mol}^{-1}$ respectively) (Figure S1) indicate stable protein-peptide complexes ${ }^{17}$. In vitro Inhibition assays were performed applying $100 \mu \mathrm{M}$ of each peptide and $10 \mu \mathrm{M}$ of SaDHNA and resulted in a non-detected activity in comparison to control assay without peptides, confirming that the predicted peptides indeed inhibit the SaDHNA thioesterase enzyme.

Fluorescence spectroscopy was applied to analyse the inhibition of SaDHNA by the designed and predicted binding peptides aforementioned. The intrinsic fluorescence quenching was assessed for a gradient concentration of Pep-1 and Pep-2. The emission spectra of SaDHNA in buffer only and SaDHNA quenched with different concentrations of Pep-1 and Pep-2 are shown in Fig. 5A and B, respectively. The amino acid sequence of SaDHNA has three tryptophan residues, W29, W79 and W129 and a 
corresponding maximum emission near $340 \mathrm{~nm}$. The gradual increase of Pep-1 to up to $1000 \mathrm{nM}$ did not shift the emission spectra maximum of SaDHNA, while Pep-2 resulted in a slight blue shift of the emission, probably explained by the slight change in the polarity of the solvent surrounding the tryptophan residues ${ }^{18}$. A decrease in the fluorescence intensity was observed for both peptides, that according to the quenching theory, may indicate a decrease in the lifetime of the excited state, corresponding to additional rate process that depopulate the excited state and/or the formation of a nonradioactive ground state between the fluorophore and the quencher resulting in non-fluorescence emission ${ }^{19,20}$.

The Stern-Volmer theory explains the relationship between fluorescence intensity and presence of a quencher; the data obtained in different concentrations of peptides were analysed independent of the quenching process, thereby the bimolecular quenching constant $\left(\mathrm{K}_{\mathrm{q}}\right)$ assigned to the efficiency of quenching and the binding or affinity constant $\left(K_{a}\right)$ for the associated complex were calculated 21,22 . SaDHNA showed a biphasic quenching behaviour, since the Stern-Volmer plot presented two linear quenching steps delimited by the red dotted line in Fig. 6A. The first one and faster quenching step was observed up to $100 \mathrm{nM}$ (left side of the red dotted line), while a second and slower quenching was observed at higher concentrations of both peptides (Fig. 6A). To investigate the quenching process and estimate $\mathrm{K}_{\mathrm{q}}$ and $\mathrm{K}_{\mathrm{a}}$ the data from the second and slower step were considered (Fig. 6B and 6C, respectively). To estimate the bimolecular quenching constant $\left(\mathrm{K}_{\mathrm{q}}\right)$, the Stern-Volmer quenching constant $\left(\mathrm{K}_{\mathrm{sv}}\right)$ was determined from the slopes of lo/I versus $\mathrm{Q}$ (quencher concentration) (Fig. 6B) and multiplied by $10^{-8}$, which corresponds approx. to the lifetime of a biomolecule fluorophore in the absence of a quenching agent ${ }^{23}$. Therefore, $\mathrm{K}_{\mathrm{q}}\left[\mathrm{M}^{-1} \mathrm{~s}^{-1}\right]$ for Pep-1 and Pep-2 were $13.3 \times 10^{12} \pm 0.6 \times 10^{12}$ and $9.3 \times$ $10^{12} \pm 1.8 \times 10^{12}$, respectively. According to the diffusion controlled quenching theory $\mathrm{K}_{\mathrm{q}}$ values close to $1 \times 10^{10} \mathrm{M}^{-1} \mathrm{~s}^{-1}$ are associated to a process involving dynamic quenchers, whereas values of $\mathrm{K}_{\mathrm{q}}$ larger than the diffusive limit indicate some type of binding interactions between the fluorophore and the quencher ${ }^{20}$. Our results demonstrated $\mathrm{K}_{\mathrm{q}}$ values 100 -fold higher than the diffusive upper limit, therefore it is most likely that the quenching process obeys the static mechanism through the complex association between SaDHNA and the designed peptides.

As the estimated $\mathrm{K}_{\mathrm{q}}$ presented higher values than expected for dynamic quenching, we analysed the data applying the static quenching theory to estimate the affinity constant $\mathrm{K}_{\mathrm{a}}$ for the associated complex. The intercept of $\log ((\mathrm{Io}-\mathrm{I}) / \mathrm{I})$ versus $\log Q$ plot (Fig. 6C) is equal to the logarithm of $\mathrm{K}_{\mathrm{a}}$, thereby the intercept antilog calculation determines the $\mathrm{K}_{\mathrm{a}}$ values for both peptides ${ }^{21}$. The respective affinity constants for Pep 1 and Pep 2 were $72.7 \pm 2.9 \mathrm{M}^{-1}$ and $3.4 \pm 0.9 \mathrm{M}^{-1}$, indicating that Pep-1 has 21 -fold higher binding affinity to SaDHNA than Pep-2. Thereby, these results strongly indicate Pep-1 as suitable candidate to be considered as lead compound for further drug development investigations, to identify compounds that can directly interact and inhibit SaDHNA, an essential and vital enzyme of the menaquinone pathway.

\section{Discussion}


We solved and refined the crystal structure of SaDHNA, and assigned SaDHNA to be a member of the HotDog fold class I superfamily of proteins, where all $\beta$-strands pointing outwards and the long main ahelix pointing towards to the core of the structure. SaDHNA associates as a dimer of homodimers, with a face-to-face (or helix-to-helix) conformation. The "HotDog" fold was first described by Leesong and coworkers for a thiol ester dehydratase-isomerase from E. coli, named FabA (pdb code 1MKA) ${ }^{24}$. Since after, a number of proteins possessing the "HotDog" fold were identified and described for several organisms ${ }^{25-29}$. Despite the absence of a consensus sequence and a sequence identity ranging only between 10 and $20 \%$, the overall fold and $\mathrm{N}$ - or C-termini secondary structure elements are similar and particular characteristic for members of the "HotDog" fold protein family. Although overall low sequence identity among all thioesterases was observed in sequence alignments, the secondary structure elements are mostly well conserved. Therefore, it is expected that the active site architecture of those enzymes remains homologous as well. Investigations involving protein superfamilies have shown that the mode of catalysis, the active site location, as well as residues involved in substrate recognition and catalysis are indeed frequently conserved among these evolutionarily related proteins ${ }^{30-32}$. This explains the relative low sequence homology between SaDHNA and other known thioesterases, even though the quaternary structures and in particular the positions of the respective active sites and residues within the interface region forming the dimers are homologous.

Thioesterases from E. coli EcYbgC as well as Haemophilus influenzae HYYbgC revealed to be more active for short acyl chain substrates, in contrast to Helicobacter pylori HpYbgC, which showed to be more active towards long acyl chains, e.g. palmitoyl- and stearoyl-Co $\mathrm{A}^{33}$. Although all enzymes possess overall the same fold, HotDog fold class I, EcYbgC, HiYbgC, HpYbgC as well as SaDHNA have some differences in their structure, which explains a divergence in the substrate specificity. In fact, we observed the presence of a long tunnel associated with the binding site of the acyl moiety of the substrate for $H p Y b g C$, which is absent in the HIYbgC. We detected a similar situation for SaDHNA, which is more active towards long acyl chains (stearoyl-CoA) in comparison to a short chain (crotonyl-CoA). The structure of SaDHNA structure reveals that activity towards long acyl chains can be associated with the presence of an extended tunnel with hydrophobic nature, involving the residues Leu35, Ile38, Tyr45; Met48, Leu122, Tyr125 and Phe126 (Figure S2), where the long acyl chain of a stearoyl-CoA may point towards this hydrophobic tunnel and is stabilized mostly through hydrophobic non-covalent interactions ${ }^{26}$.

Considering also all till now known homologous thioesterase structures, investigations performed for the native Ps4HBT and a D17N Ps4HBT mutant revealed functional residues involved in the thioesterase activity of SaDHNA. The residue His23 positioned in the N-terminus of the main a-HD helix ( $\mathbb{2}$ and a1), in substitution of Tyr24 in PS4HBT, is likely responsible for the polarization of the thioester carbonyl carbon group by a hydrogen bond with the imidazole ring of the histidine sidechain. The carbonyl sidechain from the closest residue, Asp16 (positioned within the connecting $\beta$-turn loop between $\otimes 1$ and the main $a-H D$ helix), may act as a nucleophile during the thioester bond cleavage. In fact, mutagenesis we performed for the residue Asp16 (D16A) in SaDHNA truthfully altered the catalysis rate of the SaDHNA thioesterase, resulting in a 300 -fold decrease of the hydrolysis. This important result highlights the significance of this 
particular residue for thioesterase activity of SaDHNA. Nevertheless, the mutagenesis D16A was not sufficient to completely suspend the SaDHNA thioesterase activity, showing also a divergent result compared to previously reported thioesterases ${ }^{34-36}$. This controversial result about the function of an aspartic acid in the catalysis comparing Ps4HBT, Orf6 thioesterase and SaDHNA indicates that the enzymatic mechanism of SaDHNA may not occur according to a covalent catalysis, as observed for Ps4HBT, Orf6 thioesterase but more probably by a catalysis involving a water molecule $e^{25,37,38}$. The mechanism involving the covalent catalysis is based on the proposed anhydride intermediate formation resulting from the nucleophilic attack performed by an acidic residue (aspartic or glutamic acid) in the active site, releasing the CoA thiol in the absence of a water molecule. On the other hand, the general base catalysis mechanism requires the directed activation of a water molecule which consequently acts as a nucleophile on the CoA thiol group ${ }^{39,40}$. Indeed, by a careful inspection of the native SaDHNA structure, there is a water molecule close to the proximity of BCA substrate and the sidechain of residue Asp16, inside of the tunnel of the active side, which, in fact, could support the general base mechanism in SaDHNA. Interestingly, although the mutagenesis of the aspartic acid was sufficient to completely inactivate the thioesterases from P. profundum and Pseudomonas 4HBT, for SaDHNA this mutant had substantially decreased activity, but was not fully inactivate. This surprising result indicates that SaDHNA might use the aspartic acid together with another residue as an alternative during thioesterase activity. Hydrolases, including thioesterases, frequently use the Ser-His-Asp catalytic triad to perform a bond cleavage. Within this triad, aspartic acid is an important activator during nucleophile attack, followed by serine and histidine residues. The imidazole ring of histidine possesses a $\mathrm{pK}_{\mathrm{a}}$ of approximately 6 to 7 and allows this residue to switch between protonated and unprotonated states at a physiological $\mathrm{pH}$. This individual property enables histidine to participate in general acid-base catalysis and to enhance the nucleophilicity of the hydroxyl and thiol groups. Protonated nitrogen of the imidazole ring can act as a general acid while unprotonated nitrogen acts as nucleophile, and consequently, performs as a general base ${ }^{41}$. In absence of aspartic acid in the active site, basically nitrogen from the imidazole ring of His 23 might abstract a proton of the nucleophile (a water molecule of SaDHNA native structure closed to the proximity of BCA substrate) and henceforward induce the nucleophilic attack on the carbonyl carbon of the polarized substrate (electrophile) as modelled in Figure S3. On the other hand, complementary mutagenesis we performed for the residue Glu31 (E31N) resulted in no detectable activity. The orientation of the uncharged polar sidechain of an asparagine residue might interfere with the binding of substrate in the active site, in which Glu31 is more likely to act only as a supportive residue required for the substrate binding and not being involved in the thioesterase activity. Initial evidences obtained from our investigations support the general base catalysis.

Finally, selected peptide inhibitors were successfully screened by assays and docking studies using the atomic structure of SaDHNA. In general, stable peptide-protein interactions involve hydrogen bonds, as well as complementary interactions, such as hydrophobic van der Waals interactions, leading to a high selectivity and binding affinity ${ }^{42}$. Although designed peptide inhibitors based on atomic structures have demonstrated effectiveness to inhibit bacterial protein synthesis ${ }^{43}$ and transcription ${ }^{44}$, so far, no attempt has been made to rationally design peptide inhibitors of a DHNA thioesterase. In this study we designed 
two peptide ligands. During docking analysis Pep-1 was predicted to bind inside of the SaDHNA active site, producing a stable interaction via hydrogen bonds, as well as noncovalent interactions and via aromatic ring stacking ( $\pi$ stacking), which may also contribute to the peptide stability inside the binding pocket. This stable interaction might prevent the substrate binding by blocking the active site entrance for other substrates. On the other hand, Pep-2 was predicted not to bind inside the active site, but on the surface of SaDHNA. In contrast to traditional drug target sites, e.g. enzyme active sites, protein surface regions are usually more flat and mostly have less well-defined binding pockets to bind small molecules or peptides ${ }^{45,46}$. Thoden and co-workers ${ }^{47}$ observed that the coenzyme A ribose of both 4hydroxybenzoyl-CoA substrates and the 4-hydroxyphenacyl-CoA inhibitor were positioned in a cleft located on the solvated surface of the dimer. This important observation suggests that the interaction of Pep-2 with the SaDHNA surface region might interfere with the nucleotide moiety binding of the substrate and is reflected in the overall thioesterase activity ${ }^{47,48}$. Our spectroscopy assay data obtained support the inhibitory properties of the rationally designed peptides Pep- 1 and Pep-2, which might be considered as potential lead compounds for further investigations to provide more insights about potential SaDHNA inhibition mechanisms.

\section{Conclusion}

The high-resolution structure of a 4-hydroxybenzoyl-CoA thioesterase, a key enzyme involved in the menaquinone biosynthesis pathway of $S$. aureus, resembled a Hotdog fold class I structure for the monomer. The quaternary structure of the SaDHNA homo-tetramer has four putative active sites, each located within the interface regions of two monomers, and with functional important residues Asp16, His23 from one monomer and Glu31, Tyr45 from the corresponding other monomer. Enzymatic assays and mutagenesis studies demonstrated a preference towards long chain substrates, as well as the importance of the acidic residues Asp16 and Glu31 in the active site and for substrate binding, respectively.

Specifically targeting the menaquinone synthesis pathway of antibiotic resistant bacteria, such as $S$. aureus, can be considered as a new and innovative approach for future drug development investigations to treat MRSA, due to the absence of the vitamin $\mathrm{K}$ biosynthesis in humans. Further, the peptide ligands designed for initial inhibition studies provide in this context promising information for future inhibitor development.

\section{Material And Methods}

\subsection{Cloning, expression and purification of SaDHNA}

The DHNA gene was amplified by PCR from $S$. aureus cDNA using the primers sequence presented in Supplemental Table S2. The PCR product was cloned via restriction enzyme Bsal (New England BioLabs) into $E$. coli expression vector pASK-IBA3 (IBA Lifescience) and the gene sequence was verified using automated sequencing (GATC Biotech AG, Germany). E. coli expression strain BL21 (DE3) was heat 
shock transformed with the resulting expression vector SaDHNA-IBA3 and plated on LB agar

supplemented with $100 \mathrm{mg} \cdot \mathrm{mL}^{-1}$ ampicillin. For protein expression, the cells were grown in $1 \mathrm{~L}$ of Terrific broth media supplemented with $0.4 \%(\mathrm{v} / \mathrm{v})$ glycerol (final concentration) and $100 \mathrm{mg} \cdot \mathrm{mL}^{-1}$ ampicillin at $37^{\circ} \mathrm{C}$ until reaching an optical density of 0.6 applying a wavelength of $600 \mathrm{~nm}$. Protein over-expression was induced with $200 \mathrm{ng} \mathrm{mL}^{-1}$ anhydrotetracycline (IBA Lifescience) at $37^{\circ} \mathrm{C}$ for $6 \mathrm{~h}$. Afterwards, for protein purification, the cells were harvested for $1 \mathrm{~h}$ at $4000 \times \mathrm{g}, 4^{\circ} \mathrm{C}$, and then resuspended in $100 \mathrm{mM}$ Tris- $\mathrm{HCl} \mathrm{pH} \mathrm{8.0,} 150 \mathrm{mM} \mathrm{NaCl}, 1 \mathrm{mM}$ EDTA, $1 \mathrm{mM}$ PMSF and sonicated twice for $8 \mathrm{~min}$ on ice. Soluble proteins were separated from the cell debris by centrifugation for $1 \mathrm{~h}, 18000 \times \mathrm{g}$ at $4^{\circ} \mathrm{C}$ and the supernatant was applied onto a gravity column containing Step-Tactin resin (IBA Lifescience) in the cold room. Unbound proteins were removed from the column by utilizing a washing buffer (WB) containing $100 \mathrm{mM}$ Tris-HCl pH 8.0, $150 \mathrm{mM} \mathrm{NaCl}, 1 \mathrm{mM}$ EDTA. The protein was eluted using WB supplemented with $2.5 \mathrm{mM}$ D-desthiobiotin (IBA Lifescience). Eluted protein was dialysed against $100 \mathrm{mM}$ sodium phosphate buffer pH 6.0, $100 \mathrm{mM} \mathrm{NaCl}$, applied to a pre-equilibrated HiLoad 16/600 Superdex 200 (Cytiva, former GE Healthcare) gel filtration column. After dialysis in $100 \mathrm{mM}$ sodium phosphate buffer $\mathrm{pH} 6,150 \mathrm{mM} \mathrm{NaCl}$, SaDHNA-CoA thioesterase was concentrated for crystallization experiments until 10 $\mathrm{mg} \mathrm{mL} \mathrm{mL}^{-1}$, using an extinction coefficient of $41370 \mathrm{M}^{-1} \mathrm{~cm}^{-1}$, provided by Protparam program (http://web.expasy.org/protparam/).

\subsection{Thioesterase activity assay}

The thioesterase activity of SaDHNA was measured according to a protocol of Rodríguez-Guilbe and coworkers $^{36}$. In a ELISA microplate the formation of 2-nitro-5-thiobenzoate anion (TNB2-) by the reaction of thiolate anion $\left(\mathrm{RS}^{-}\right)$with Ellman's reagent $\left(\mathrm{DTNB}^{2-}\right)$ and one mixed disulphide (R-S-TNB ${ }^{-}$) catalysed by purified SaDHNA wild type (WT), D16A and E31N was followed by monitoring the change in absorbance of thionitrobenzoic acid (TNB) at $412 \mathrm{~nm}$ (extinction coefficient of $13.600 \mathrm{M}^{-1} \mathrm{~cm}^{-1}$ ) using a TECAN GENios plate reader (XFLUOR4 Version: V 4.40, MTX Lab Systems, Inc, USA). The enzymatic assays were performed in a total volume of $200 \mu \mathrm{l}$ at room temperature by incubating $10 \mu \mathrm{M}$ of enzyme in $50 \mathrm{mM}$

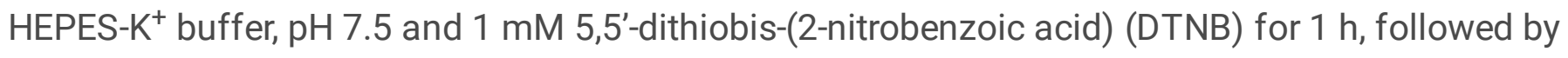
addition of $100 \mu \mathrm{M}$ stearoyl-CoA ("long chain") or $1 \mathrm{mM}$ crotonyl-CoA ("short chain"). All enzymatic assays were carried out in triplicates, from individual protein production and purification batches. Peptide binding- and inhibition assays of SaDHNA were performed according to the before mentioned thioesterase activity assay, applying a constant peptide concentration of $100 \mu \mathrm{M}$. Control reactions were performed with the same reagents, without adding the substrate in order to detect nonspecific conversion of DTNB by the enzyme. In addition, uncatalyzed reaction rates were monitored by the combination of same reagents without the enzyme.

\subsection{Site-directed Mutagenesis}

In order to obtain more information regarding the putative interactions identified for D16 and E31 and the substrate benzoyl-CoA obtained by superimposition using the homologous structure of PS4HBT (pdb code 1LO9), as well as to obtain more insights about the structure-function relationship of SaDHNA, site- 
directed mutagenesis for the residues D16 and E31 was performed by plasmid PCR amplification, according to Edelheit (2009) ${ }^{49}$. Corresponding PCR was performed by amplification of the parental plasmid DNA in two PCR tubes and adding the forward or the reverse primer, as listed in supplemental table S2, using the Q5 High fidelity DNA polymerase (New England Biolabs). After PCR, the reaction product was combined into one single tube, denaturated by heat to separate the recently synthesized DNA strain from the template and cooled down gradually to allow the annealing of the complementary chains. The original DNA template was digested by adding the restriction enzyme Dnpl (Thermo Fisher Scientifc) and as a final step, used to transform BL21 (DE3) competent cells. Sanger sequencing (GATC Biotech AG, Germany) was performed to verify the sequence of the purified DNA plasmids.

\subsection{Crystallization, heavy atom derivative, data collection and structure determination}

X-ray suitable crystals of SaDHNA were obtained applying the sitting drop vapor-diffusion technique mixing in a ratio of $1: 1$ protein solution $\left(10 \mathrm{mg} \cdot \mathrm{mL}^{-1}\right)$ and reservoir solution consisting of $100 \mathrm{mM}$ HEPES-Na ${ }^{+} \mathrm{pH}$ 7.0, 1.0 M lithium sulphate, equilibrated against $300 \mu \mathrm{L}$ of reservoir solution utilizing MRC Maxi 48-wells plates (Molecular dimensions, UK) at $293 \mathrm{~K}$. For cryo-cooling prior to X-ray data collection crystals were transferred to a new reservoir solution containing $15 \%(\mathrm{v} / \mathrm{v})$ glycerol and flash-cooled in a nitrogen stream at $100 \mathrm{~K}$. Diffraction data for native SaDHNA were collected at the EMBL beamline P13 at PETRA III (DESY, Hamburg). In order to obtain phase information, heavy atom derivative soaking was performed using a final concentration of $1.25 \mathrm{mM}$ potassium tetrachloroplatinate II (Hampton Research, USA), added to the crystal droplet $24 \mathrm{~h}$ before X-ray data collection. A platinum-SAD single-wavelength anomalous dispersion/diffraction (SAD) dataset was collected at $1.072 \AA$ wavelength and up to $2.0 \AA$ resolution at the EMBL beamline P14 at PETRA III (DESY, Hamburg) and was used for heavy atom localization and subsequent phasing. SaDHNA and derivative data were indexed, integrated using the XDS software package ${ }^{50}$ and were scaled using the program AIMLESS from the CCP4i software suite ${ }^{51-}$

53. In order to obtain phase information, the EMBL-HH Automated Crystal Structure Determination Platform Auto Rickshaw (EMBL Hamburg, Germany) (www.embl-hamburg.de/Auto-Rickshaw/) ${ }^{54}$ was used. Afterwards, successive rounds of model building and refinement were performed using the program REFMAC5, version $5.8 .0131^{55}$ from CCP4i and the program Coot version $0.8 .1^{56}$ for model building. All structure figures were generated using the PyMOL software suite version 1.3 and the final SaDHNA structure was deposited at the Protein Data Bank (pdb code 6FDG).

\subsection{Small Angle X-ray Scattering (SAXS) measurements}

Small-angle X-ray scattering data of monodispers SaDHNA at concentrations from 0.8 to $2.5 \mathrm{mg} \mathrm{ml}^{-1}$ were collected at EMBL beamline P12 at the storage ring PETRA III (DESY, Hamburg, Germany). The monodispersity of the sample solutions was verified prior to SAXS data collection applying DLS using a SpectroLight 300 instrument (Xtal Concepts, Germany). Protein was applied in $100 \mathrm{mM}$ sodium phosphate buffer $\mathrm{pH} 6.0$ and $150 \mathrm{mM} \mathrm{NaCl}$ with a sample volume of $25 \mu \mathrm{l}$ at $10^{\circ} \mathrm{C}$. SAXS data were collected at a sample-detector distance of $3.1 \mathrm{~m}$, a wavelength of $\lambda=0.124 \mathrm{~nm}$ viand applying a $2 \mathrm{D}$ 
photon-counting Pilatus $2 \mathrm{M}$ pixel detector (Dectris) with the momentum transfer ranging from $0.03 \mathrm{~nm}^{-1}$ $<\mathrm{s}<5 \mathrm{~nm}^{-1}$ ( $\mathrm{s}=4 \pi \sin \theta \lambda^{-1}$, where $2 \theta$ is the scattering angle). Data were normalized to the intensity of the transmitted beam and radially averaged. Scattering amplitudes from 20 successive X-ray exposures of $45 \mathrm{~ms}$ each were averaged and subtracted from the average of 40 buffer exposures. The Guinier region, radius of gyration $R_{g}$ and the particle pair-distance distribution function $p(r)$, which further provides the maximum dimension $D_{\max }$ of the protein, were obtained and evaluated applying the program PRIMUSQT, part of the ATSAS software suite ${ }^{57}$. Low-resolution chain-like ab initio shapes of SaDHNA showing tetramer symmetry were subsequently generated using the composite scattering curves applying the program $\mathrm{GASBOR}^{58}$ and using a total number of 620 amino acid dummy spheres and 611 water molecules.

\subsection{Docking investigations, peptide rational design and synthesis}

Docking studies were carried out with SaDHNA homodimer using the BioLuminate software from the Schrödinger suite (Schrödinger, LLC, New York, 2021). The peptides were designed based on the structure of the natural substrate 1,4-dihydroxy-2-naphthoyl-CoA and used for peptide docking calculation applying the BioLuminate tool using the Molecular Mechanics/Generalized Born Surface Area (MM/GBSA) method to calculate the free binding energy ${ }^{59}$. Afterwards, peptides, Pep-1 and Pep-2, with the lowest scores obtained from the calculated free energy binging were synthesized and used for further in vitro inhibition assays.

\subsection{Investigation inhibitory activity of specific peptides Sa using fluorescence spectrophotometry}

Fluorescence measurements were performed using a Cary eclipse fluorescence spectrophotometer coupled with a peltier temperature control (Agilent, USA). All assays were carried out in $1 \mathrm{~mL}$ cuvettes at $22^{\circ} \mathrm{C}$ with excitation/emission slits at $20 \mathrm{~nm}$ each. The applied excitation wavelength was $290 \mathrm{~nm}$ and emission spectra were collected in the range of 300 up to $420 \mathrm{~nm}$ in increments of $1 \mathrm{~nm}$. SaDHNA quenching experiments were performed with two peptides (Pep-1: YGSDGR and Pep-2: EGEYE, using a concentration range from 60 up to $1000 \mathrm{nM}$. SaDHNA at a concentration of $60 \mathrm{nM}$ in $100 \mathrm{mM}$ sodium phosphate buffer $\mathrm{pH} 6.0,150 \mathrm{mM} \mathrm{NaCl}$ was applied and small volumes of the stock peptide solution were sequentially added to the cuvette for quenching analysis. Each SaDHNA-peptide assay was performed five times. As a control the fluorescence of all buffer and all peptide solutions were measured to correct the observed fluorescence accordingly. The quenching process was assessed by the SternVolmer theory ${ }^{60}$.

\section{Declarations}

\section{Conflict of interest}


The authors declare that there are no competing interests.

\section{Acknowledgement and funding}

This work was supported by Coordenação de Aperfeiçoamento Pessoal de Ensino Superior (CAPES), Brazil. Further, the authors would like to thank the Fundação de Amparo à Pesquisa do Estado de São Paulo (FAPESP) (grants 2015/26722-8, 2017/03966-4 and 2012/12790-3). And the authors acknowledge financial support obtained from the Cluster of Excellence 'Advanced Imaging of Matter' of the Deutsche Forschungsgemeinschaft (DFG) - EXC 2056 - project ID 390715994, from the Helmholtz Excellence Network 'Structure, Dynamics and Control on the Atomic Scale', from BMBF via projects, 05K19GU4, 05K20GUB and support obtained from the Joachim-Herz-Stiftung Hamburg via the project InfectoPhysics and financial support via the collaborative project between Universities São Paulo (USP) and Hamburg (UHH), the UHH-USP-FAPESP Sprint Project 2019.

\section{Author contributions}

A.M.M., H.B., S.F., C.W. and C.B. designed the experiment(s), A.M.M., H.B., J.L., M.P., C.M., S.F and R.S. conducted the experiment(s), A.M.M., H.B., S.F., C.W. and C.B analysed the results. All authors reviewed the manuscript.

\section{References}

1. Abbasi, S. H., Aftab, R. A. \& Chua, S. S. Risk factors associated with nosocomial infections among end stage renal disease patients undergoing hemodialysis: A systematic review. PLoS One 15, e0234376-e0234376 (2020).

2. Turner, N. A. et al. Methicillin-resistant Staphylococcus aureus: an overview of basic and clinical research. Nat. Rev. Microbiol. 17, 203-218 (2019).

3. Essack, S. Y. The Development of $\beta$-Lactam Antibiotics in Response to the Evolution of $\beta$ Lactamases. Pharm. Res. 18, 1391-1399 (2001).

4. Koch, G. et al. Evolution of resistance to a last-resort antibiotic in Staphylococcus aureus via bacterial competition. Cell 158, 1060-1071 (2014).

5. Venter, H. Reversing resistance to counter antimicrobial resistance in the World Health Organisation's critical priority of most dangerous pathogens. Biosci. Rep. 39, BSR20180474 (2019).

6. Kurosu, M. \& Begari, E. Vitamin $\mathrm{K}_{2}$ in electron transport system: are enzymes involved in vitamin $\mathrm{K}_{2}$ biosynthesis promising drug targets? Molecules 15, 1531-1553 (2010).

7. Priyadarshi, A. et al. Structural insights of the MenD from Escherichia coli reveal ThDP affinity. Biochem. Biophys. Res. Commun. 380, 797-801 (2009).

8. Truglio, J. J. et al. Crystal Structure of Mycobacterium tuberculosis MenB, a Key Enzyme in Vitamin $\mathrm{K}_{2}$ Biosynthesis. J. Biol. Chem. 278, 42352-42360 (2003). 
9. Benning, M. M. et al. The three-dimensional structure of 4-hydroxybenzoyl-CoA thioesterase from Pseudomonas sp. strain CBS-3. J. Biol. Chem. 273, 33572-33579 (1998).

10. Matarlo, J. S. et al. Mechanism of MenE inhibition by acyl-adenylate analogues and discovery of novel antibacterial agents. Biochemistry 54, 6514-6524 (2015).

11. Choi, S.-R., Frandsen, J. \& Narayanasamy, P. Novel long-chain compounds with both immunomodulatory and MenA inhibitory activities against Staphylococcus aureus and its biofilm. Sci. Rep. 7, 40077 (2017).

12. Krissinel, E. \& Henrick, K. Inference of Macromolecular Assemblies from Crystalline State. J. Mol. Biol. 372, 774-797 (2007).

13. Jo, S., Vargyas, M., Vasko-Szedlar, J., Roux, B. \& Im, W. PBEQ-Solver for online visualization of electrostatic potential of biomolecules. Nucleic Acids Res. 36, W270-W275 (2008).

14. Im, W., Beglov, D. \& Roux, B. Continuum solvation model: Computation of electrostatic forces from numerical solutions to the Poisson-Boltzmann equation. Comput. Phys. Commun. 111, 59-75 (1998).

15. McWilliam, H. et al. Analysis Tool Web Services from the EMBL-EBI. Nucleic Acids Res. 41, 597-600 (2013).

16. Thoden, J. B., Holden, H. M., Zhuang, Z. \& Dunaway-Mariano, D. X-ray crystallographic analyses of inhibitor and substrate complexes of wild-type and mutant 4-Hydroxybenzoyl-CoA thioesterase. J. Biol. Chem. 277, 27468-27476 (2002).

17. Shivakumar, D., Harder, E., Damm, W., Friesner, R. A. \& Sherman, W. Improving the prediction of absolute solvation Free Energies using the Next Generation OPLS Force Field. J. Chem. Theory Comput. 8, 2553-2558 (2012).

18. Royer, C. A. Probing protein folding and conformational transitions with fluorescence. Chem. Rev. 106, 1769-1784 (2006).

19. Chen, Y. \& Barkley, M. D. Toward understanding tryptophan fluorescence in proteins. Biochemistry 37, 9976-9982 (1998).

20. Albrecht, C. Joseph R. Lakowicz: Principles of fluorescence spectroscopy, 3rd Edition. Anal. Bioanal. Chem. 390, 1223-1224 (2008).

21. Boukari, I., O’Donohue, M., Rémond, C. \& Chabbert, B. Probing a family GH11 endo- $\beta-1,4-x y l a n a s e$ inhibition mechanism by phenolic compounds: Role of functional phenolic groups. J. Mol. Catal. $B$ Enzym. 72, 130-138 (2011).

22. Silva, D., Cortez, C. M. \& Louro, S. R. W. Quenching of the intrinsic fluorescence of bovine serum albumin by chlorpromazine and hemin. Brazilian J. Med. Biol. Res. 37, 963-968 (2004).

23. Valensin, G., Kushnir, T. \& Navon, G. Selective and nonselective proton spin-lattice relaxation studies of enzyme-substrate interactions. J. Magn. Reson. 46, 23-29 (1982).

24. Leesong, M., Henderson, B. S., Gillig, J. R., Schwab, J. M. \& Smith, J. L. Structure of a dehydrataseisomerase from the bacterial pathway for biosynthesis of unsaturated fatty acids: Two catalytic 
activities in one active site. Structure 4, 253-264 (1996).

25. Cao, J., Xu, H., Zhao, H., Gong, W. \& Dunaway-Mariano, D. The mechanisms of human Hotdog-fold thioesterase 2 (hTHEM2) substrate recognition and catalysis illuminated by a structure and function based analysis. Biochemistry 48, 1293-1304 (2009).

26. Pidugu, L. S., Maity, K., Ramaswamy, K., Surolia, N. \& Suguna, K. Analysis of proteins with the 'hot dog' fold: Prediction of function and identification of catalytic residues of hypothetical proteins. BMC Struct. Biol. 9, 37 (2009).

27. Widhalm, J. R., van Oostende, C., Furt, F. \& Basset, G. J. C. A dedicated thioesterase of the Hotdogfold family is required for the biosynthesis of the naphthoquinone ring of vitamin $\mathrm{K}_{1}$. Proc. Natl. Acad. Sci. 106, 5599 LP - 5603 (2009).

28. Gonzalez, C. F. et al. Structure and activity of the Pseudomonas aeruginosa hotdog-fold thioesterases PA5202 and PA2801. Biochem. J. 444, 445-455 (2012).

29. Ismail, W. Benzoyl-coenzyme A thioesterase of Azoarcus evansii: properties and function. Arch. Microbiol. 190, 451-460 (2008).

30. Todd, A. E., Orengo, C. A. \& Thornton, J. M. Evolution of function in protein superfamilies, from a structural perspective. J. Mol. Biol. 307, 1113-1143 (2001).

31. Gerlt, J. A. \& Babbitt, P. C. Divergent evolution of enzymatic function: Mechanistically diverse superfamilies and functionally distinct suprafamilies. Annu. Rev. Biochem. 70, 209-246 (2001).

32. Glasner, M. E., Gerlt, J. A. \& Babbitt, P. C. Mechanisms of protein evolution and their application to protein engineering. in Advances in Enzymology and Related Areas of Molecular Biology 193-239 (John Wiley \& Sons, Ltd, 2010). doi:10.1002/9780471224464.ch3.

33. Angelini, A., Cendron, L., Goncalves, S., Zanotti, G. \& Terradot, L. Structural and enzymatic characterization of HP0496, a YbgC thioesterase from Helicobacter pylori. Proteins Struct. Funct. Genet. 72, 1212-1221 (2008).

34. Zhuang, Z. et al. Kinetic, Raman, NMR, and site-directed mutagenesis studies of the Pseudomonas sp. strain CBS3 4-hydroxybenzoyl-CoA thioesterase active site. Biochemistry 41, 11152-11160 (2002).

35. Zhuang, Z. et al. Investigation of the catalytic mechanism of the hotdog-fold enzyme superfamily Pseudomonas sp. strain CBS3 4-hydroxybenzoyl-CoA thioesterase. Biochemistry 51, 786-794 (2012).

36. Rodríguez-Guilbe, M., Oyola-Robles, D., Schreiter, E. R. \& Baerga-Ortiz, A. Structure, activity, and substrate selectivity of the Orf6 thioesterase from Photobacterium profundum. J. Biol. Chem. 288, $10841-10848$ (2013).

37. Kotaka, M. et al. Structure and catalytic mechanism of the thioesterase CalE7 in enediyne biosynthesis. J. Biol. Chem. 284, 15739-15749 (2009).

38. Kunishima, N. et al. A novel induced-fit reaction mechanism of asymmetric hot dog thioesterase Paal. J. Mol. Biol. 352, 212-228 (2005). 
39. Song, F. et al. The catalytic mechanism of the hotdog-fold enzyme superfamily 4-hydroxybenzoylCoA thioesterase from Arthrobactersp. strain SU. Biochemistry 51, 7000-7016 (2012).

40. Sun, Y. et al. Molecular Basis of the General Base Catalysis of an a/ $\beta$-Hydrolase Catalytic Triad. J. Biol. Chem. 289, 15867-15879 (2014).

41. Ingle, R. A. Histidine biosynthesis. Arab. B. 9, e0141-e0141 (2011).

42. Kaspar, A. A. \& Reichert, J. M. Future directions for peptide therapeutics development. Drug Discov. Today 18, 807-817 (2013).

43. Mardirossian, M. et al. Peptide inhibitors of bacterial protein synthesis with broad spectrum and SbmA-independent bactericidal activity against clinical pathogens. J. Med. Chem. 63, 9590-9602 (2020).

44. Kaur, G., Kapoor, S., Kaundal, S., Dutta, D. \& Thakur, K. Structure-guided designing and evaluation of peptides targeting bacterial transcription. Front. Bioeng. Biotechnol. 8, 797 (2020).

45. Ran, X. \& Gestwicki, J. E. Inhibitors of protein-protein interactions (PPIs): an analysis of scaffold choices and buried surface area. Curr. Opin. Chem. Biol. 44, 75-86 (2018).

46. Cunningham, A. D., Qvit, N. \& Mochly-Rosen, D. Peptides and peptidomimetics as regulators of protein-protein interactions. Curr. Opin. Struct. Biol. 44, 59-66 (2017).

47. Thoden, J. B., Holden, H. M., Zhuang, Z. \& Dunaway-Mariano, D. X-ray crystallographic analyses of inhibitor and substrate complexes of wild-type and mutant 4-hydroxybenzoyl-CoA thioesterase. J. Biol. Chem. 277, 27468-27476 (2002).

48. Obarska-Kosinska, A., lacoangeli, A., Lepore, R. \& Tramontano, A. PepComposer: computational design of peptides binding to a given protein surface. Nucleic Acids Res. gkw366 (2016) doi:10.1093/nar/gkw366.

49. Edelheit, O., Hanukoglu, A. \& Hanukoglu, I. Simple and efficient site-directed mutagenesis using two single-primer reactions in parallel to generate mutants for protein structure-function studies. BMC Biotechnol. 9, 61 (2009).

50. Kabsch, W. XDS. Acta Crystallogr. D. Biol. Crystallogr. 66, 125-32 (2010).

51. Evans, P. R. \& Murshudov, G. N. How good are my data and what is the resolution? Acta Crystallogr. Sect. D 69, 1204-1214 (2013).

52. Winn, M. D. et al. Overview of the CCP4 suite and current developments. Acta Crystallographica Section D: Biological Crystallography vol. 67 235-242 (2011).

53. Potterton, E., Briggs, P., Turkenburg, M. \& Dodson, E. A graphical user interface to the CCP4 program suite. Acta Crystallogr. - Sect. D Biol. Crystallogr. 59, 1131-1137 (2003).

54. Panjikar, S., Parthasarathy, V., Lamzin, V. S., Weiss, M. S. \& Tucker, P. A. Auto-Rickshaw. an automated crystal structure determination platform as an efficient tool for the validation of an X-ray diffraction experiment. Acta Crystallogr. Sect. D 61, 449-457 (2005).

55. Murshudov, G. N., Vagin, A. A. \& Dodson, E. J. Refinement of macromolecular structures by the Maximum-Likelihood method. Acta Crystallogr. Sect. D 53, 240-255 (1997). 
56. Emsley, P., Lohkamp, B., Scott, W. G. \& Cowtan, K. Features and development of Coot. Acta Crystallogr. Sect. D 66, 486-501 (2010).

57. Franke, D. et al. ATSAS 2.8: a comprehensive data analysis suite for small-angle scattering from macromolecular solutions. J. Appl. Crystallogr. 50, 1212-1225 (2017).

58. Svergun, D. I., Petoukhov, M. V \& Koch, M. H. J. Determination of domain structure of proteins from Xray solution scattering. Biophys. J. 80, 2946-2953 (2001).

59. Genheden, S. \& Ryde, U. The MM/PBSA and MM/GBSA methods to estimate ligand-binding affinities. Expert Opin. Drug Discov. 10, 449-61 (2015).

60. Gehlen, M. H. The centenary of the Stern-Volmer equation of fluorescence quenching: From the single line plot to the SV quenching map. J. Photochem. Photobiol. C Photochem. Rev. 42, 100338 (2020).

\section{Figures}



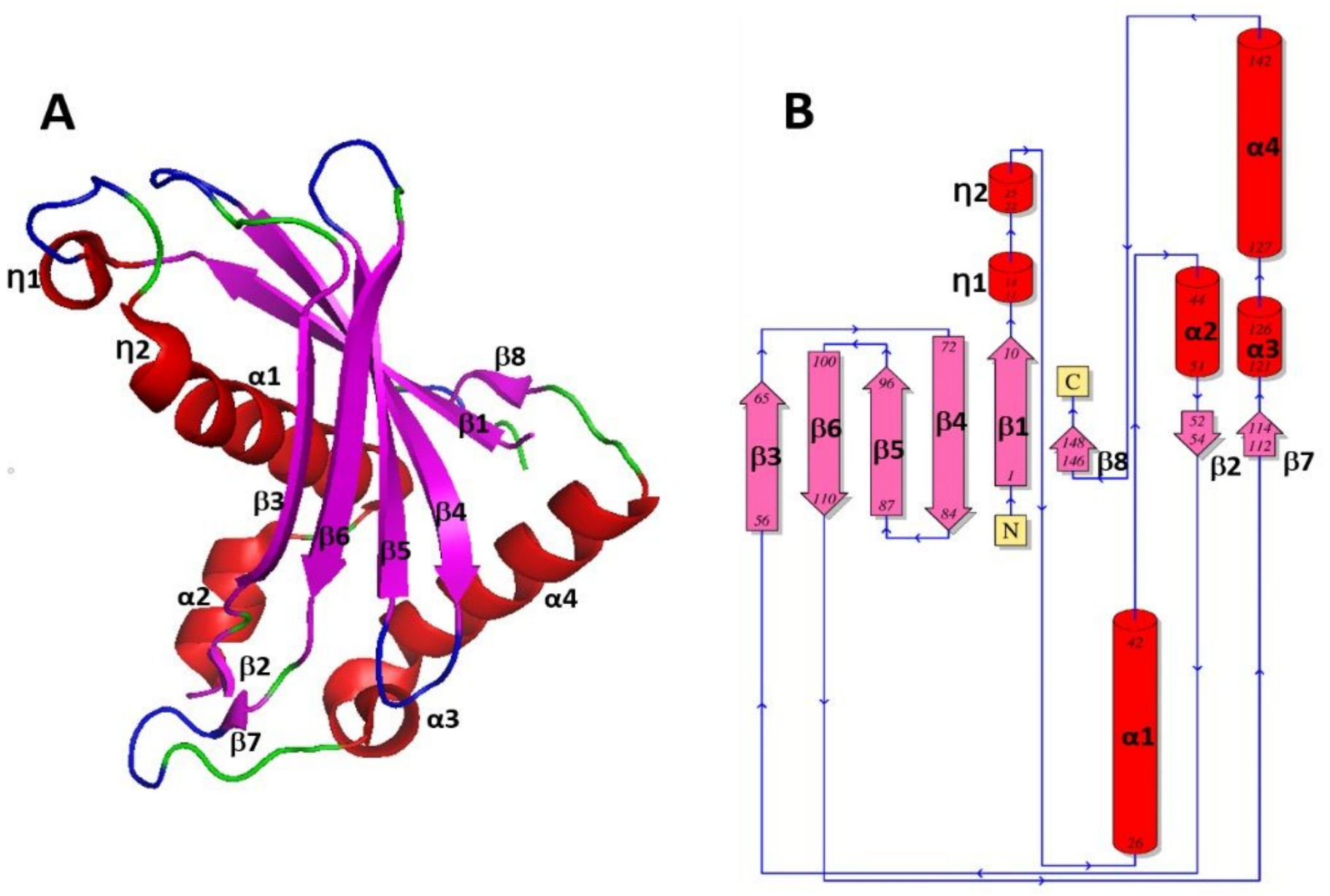

C
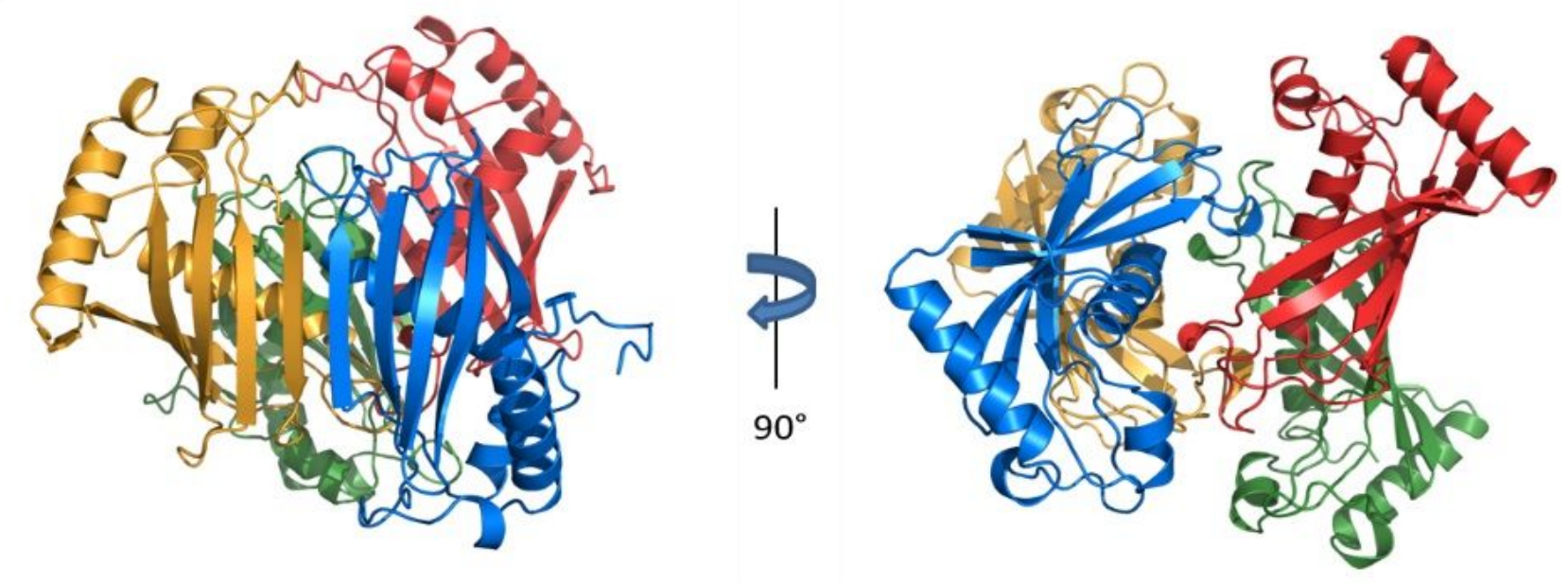

Figure 1

(A) Cartoon representation of the SaDHNA monomer. (B) The secondary structure of SaDHNA is shown schematically and annotated including the topology plot of its HotDog domain. (C) The quaternary arrangement of SaDHNA is shown for the individual monomer chains with chain A in blue, B in yellow, $C$ in red and $D$ in green. The figure was created applying the program PyMOL (Molecular Graphics System, Version 1.0.5.4 Schrödinger, LLC). 
A
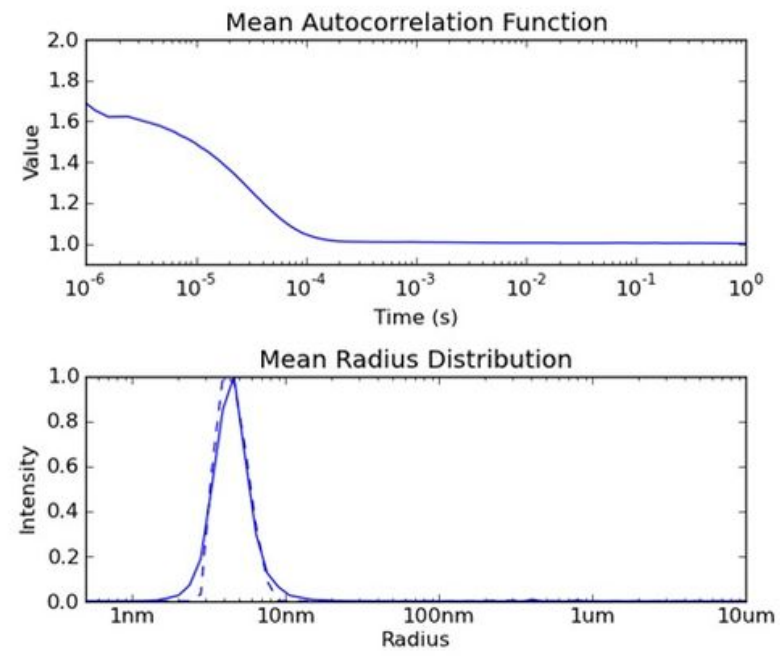

C

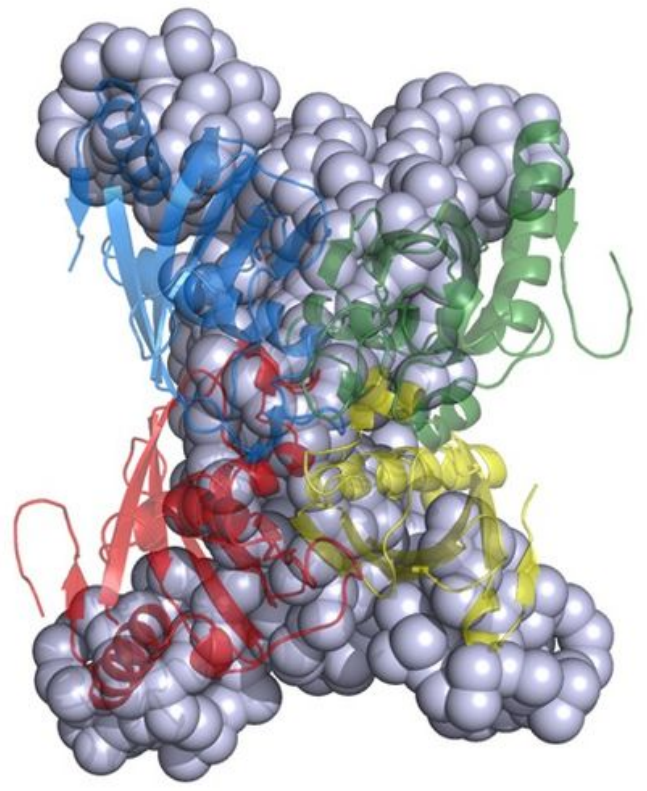

B
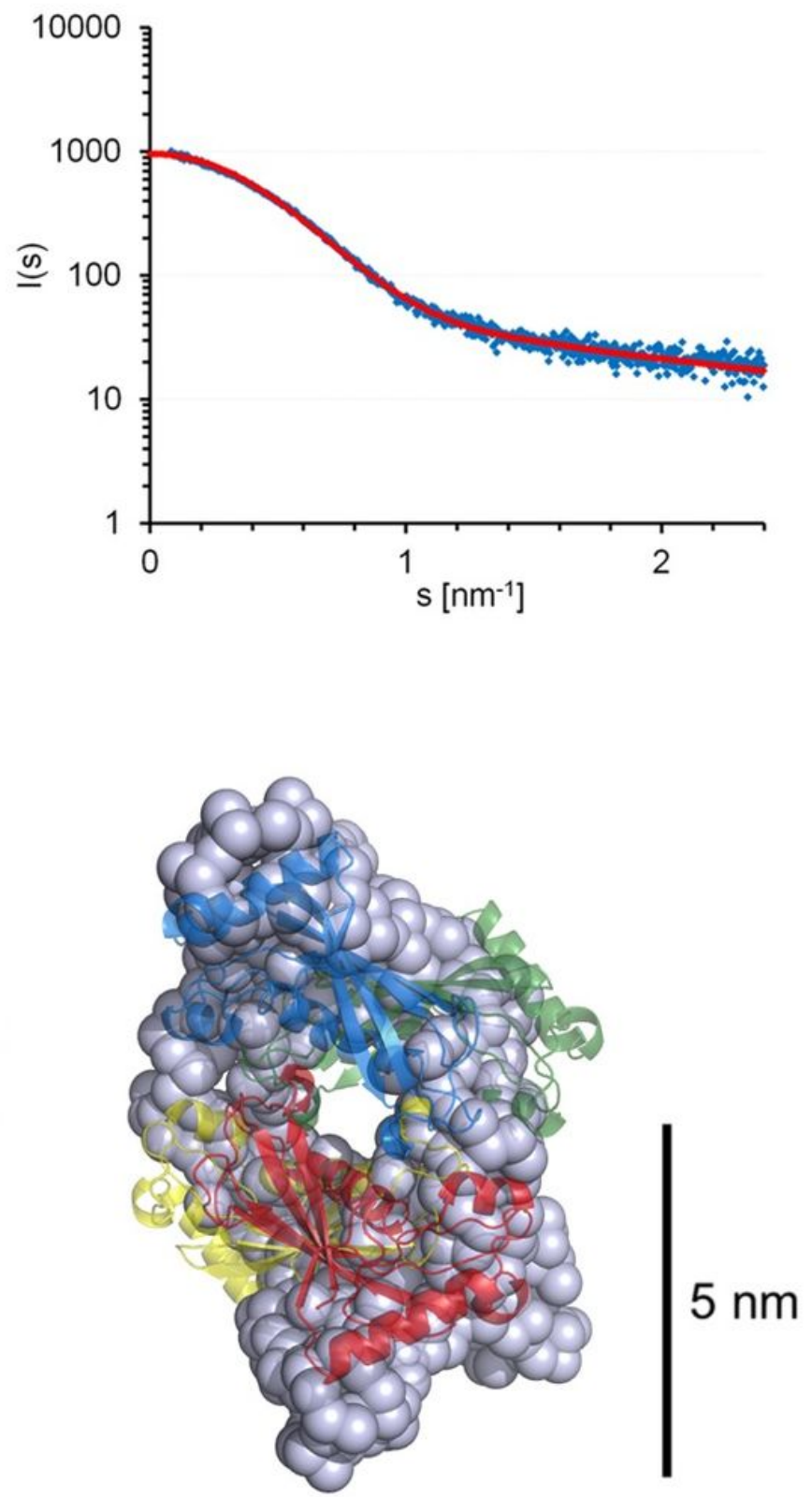

\section{Figure 2}

DLS and SAXS data of SaDHNA. (A) Autocorrelation function and corresponding mean radius distribution obtained by DLS, averaged over $300 \mathrm{~s}$. (B) Averaged X-ray scattering intensities of SaDHNA (blue dots) in arbitrary units plotted against the scattering angle, i.e. momentum transfer values ranging from $\mathrm{s}=0.05$ to $2.4 \mathrm{~nm}-1$. The red fit function corresponds to the SaDHNA ab initio model displayed in figure panel $\mathrm{C}$, sharing a $\times 2$-value of 1.54 with the corresponding experimental scattering data. (C) Superimposition of a single SaDHNA ab initio model calculated applying the program GASBOR and the tetramer of SaDHNA revealed by the crystal structure. 


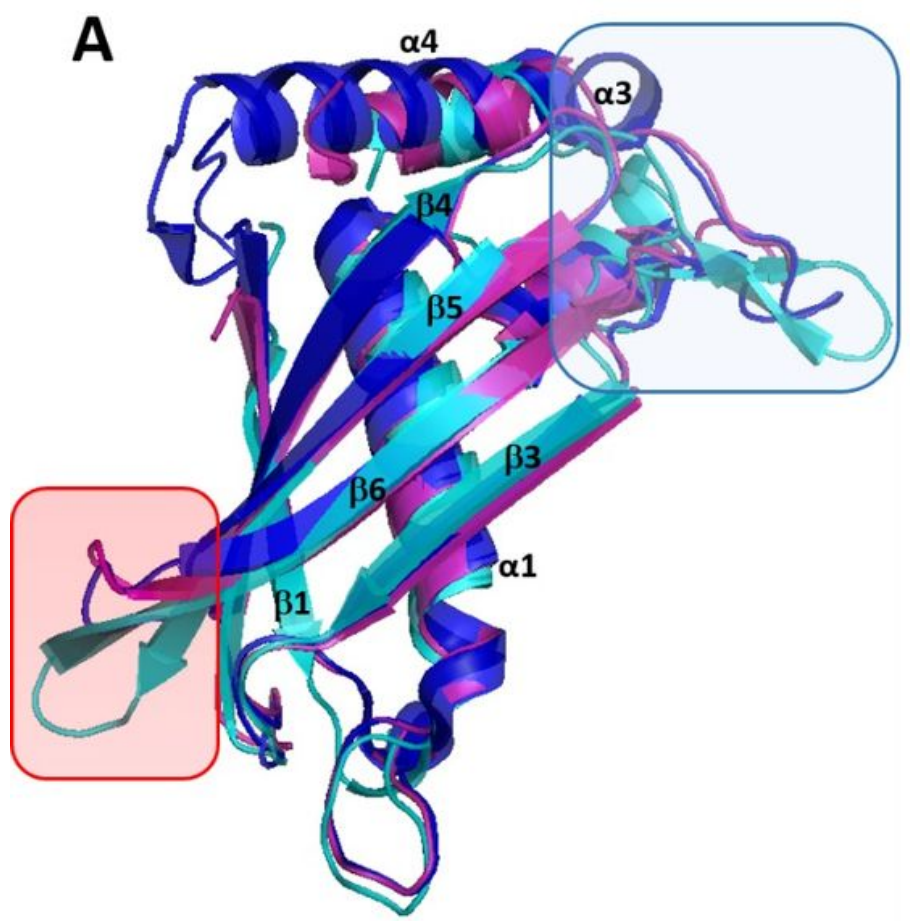

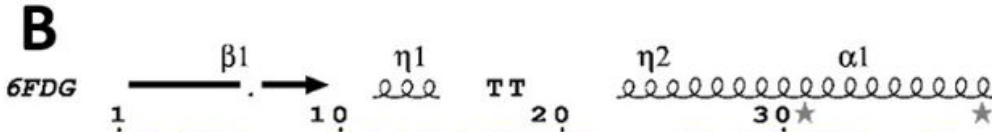

6FDG MIYSIT. EIEARYAETDKMGVIYHGNYATWFEVRLDYIS

$1254 . M E S V T$. RIKVR Y A T T QMGVVHH SVYAVY L E AARVDF LE

1BVQ MARSITMQQRIEEGDCDPA.GIVWYPNYHRWLDAASRNYF I

6FDG $\underset{40}{\text { el }} \stackrel{\alpha 2}{\text { eelee }} \underset{50}{\stackrel{\beta 2}{\rightarrow}} \frac{\beta 3}{60 \star \star}$

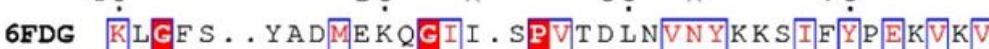

1254 RAGLP.. YHRVEARGVF . FPVVELGITFRAPARFGEVVEV

1BVQ KCGLPPWRQTVVERGIVGTPIVSCNASEVCTASYDVITI

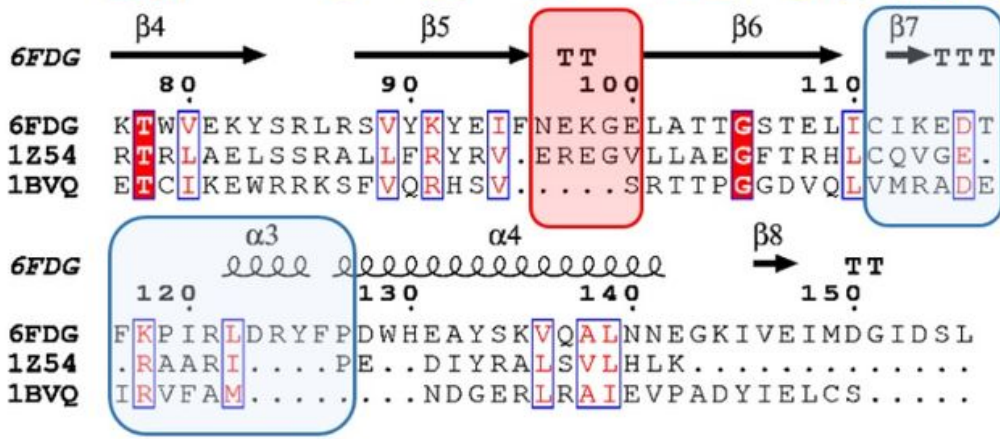

Figure 3

(A) Superimposition of SaDHNA (dark blue) with homologous structures, PDB codes 1Z54 of T. thermophylus (pink) and 1BVQ of Pseudomonas sp (cyan). (B) Protein sequence alignment. The light blue and red boxes illustrate the highest variability compared between the homologue enzymes. Multiple sequence alignment was performed applying the program ClustalOmega15 with default parameters.

A

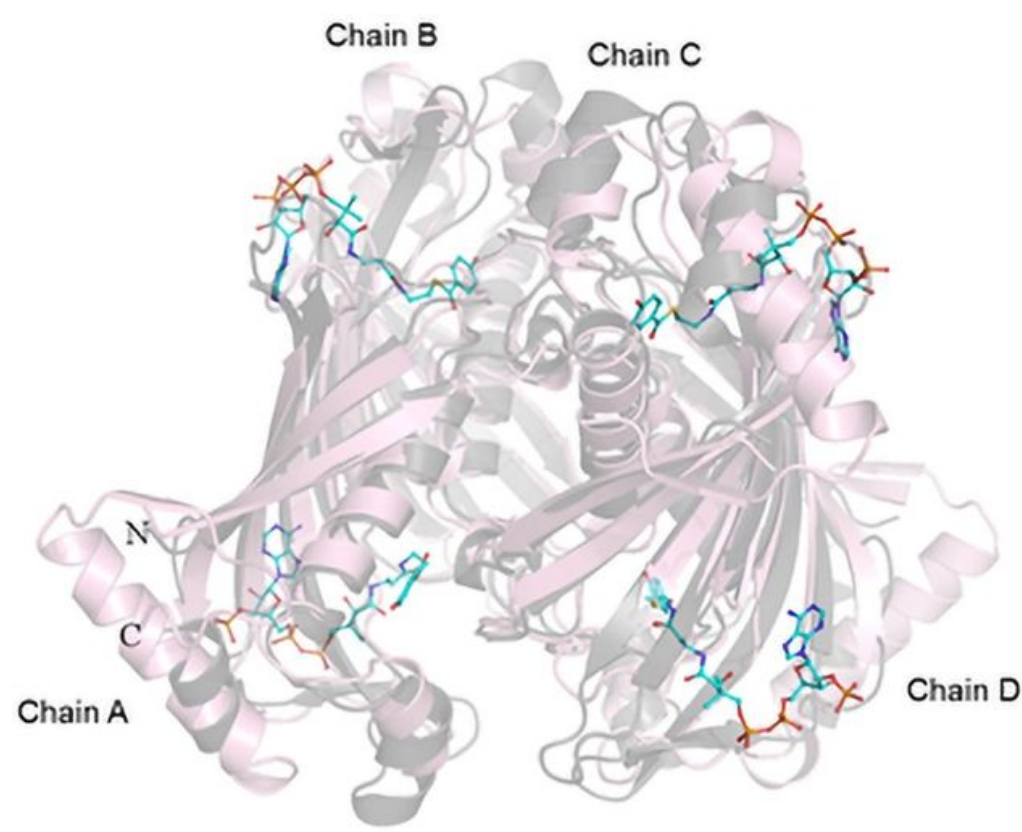

B

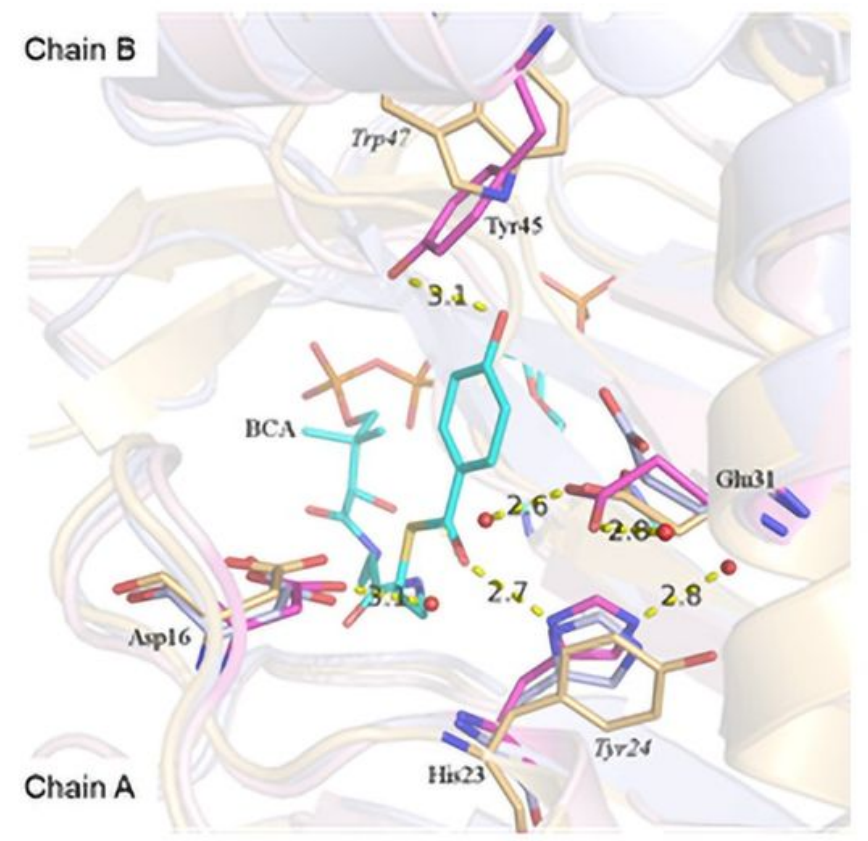

Figure 4 
Putative active sites of the tetrameric SaDHNA-CoA thioesterase. (A) Ribbon representation of SaDHNA (light pink) superimposed with the 4-hydroxybenzoyl-CoA thioesterase D17N mutant structure of Pseudomonas (PDB code 1LO9) (light grey). The substrate 4-hydroxybenzoyl-CoA (BCA) complexed with the D17N Ps4HBT mutant is shown in stick representation; carbon in cyan; sulfur in yellow; phosphate in orange, nitrogen in blue, oxygen in red; " $\mathrm{N}$ " and " $\mathrm{C}$ " indicate $\mathrm{N}$ - and $\mathrm{C}$-terminus, respectively. (B) Residues at the proposed active site region with assigned thioesterase activity of SaDHNA (pink), Pseudomonas 4HBT structure (yellow) and hypothetical Thioesterase from Thermus thermophilus (pdb code 1Z54) (light gray). Italic amino acid residue labels indicate corresponding residues in the Pseudomonas 4HBT structure (yellow).
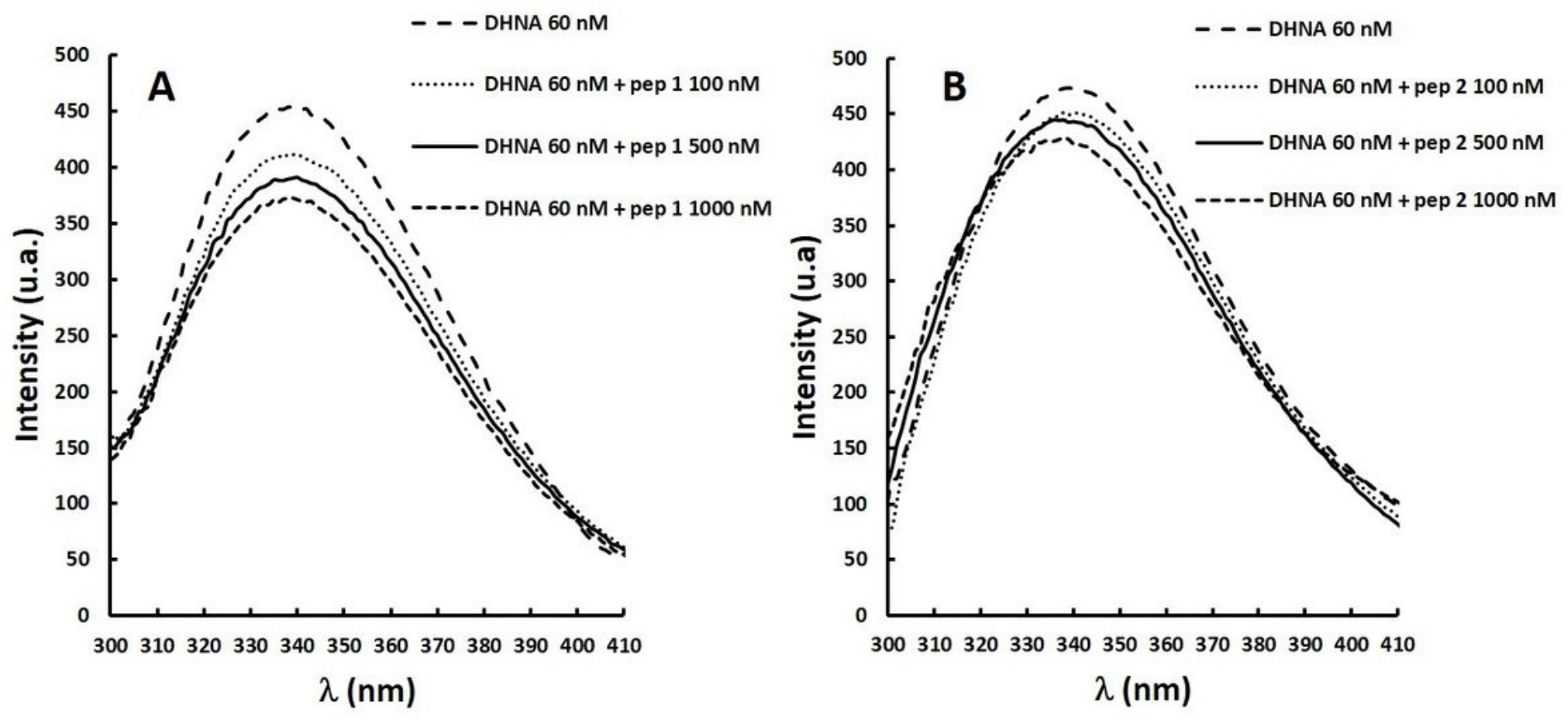

\section{Figure 5}

Fluorescence emission spectra of SaDHNA (60 nM) in the absence and the presence of the gradual concentrations of (A) Pep-1 and (B) Pep-2 up to $1000 \mathrm{nM}$.
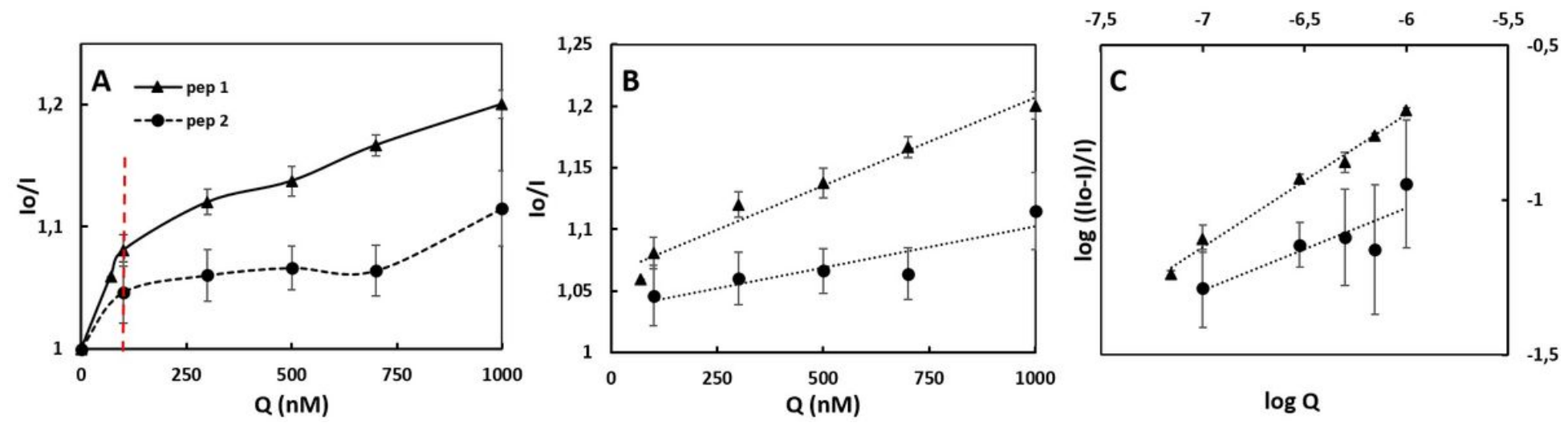

Figure 6 
(A) Stern-Volmer plot for Pep-1 and Pep-2 ranging from 0 to 1000 nM. (B) Stern-Volmer plot indicating the bimolecular quenching constant (Kq). (C) Static quenching plot to estimate the affinity-binding constant $(\mathrm{Ka})$.

\section{Supplementary Files}

This is a list of supplementary files associated with this preprint. Click to download.

- SupplementarymateriaISaDHNA25.10.2021.docx 